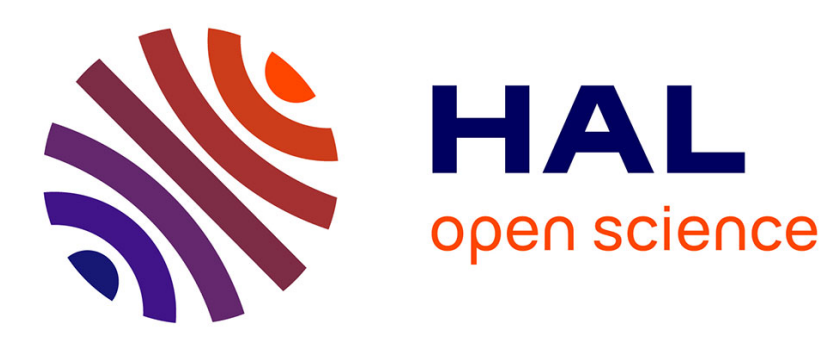

\title{
Utilisation des micropsychromètres pour la mesure du potentiel hydrique du sol en laboratoire et in situ
}

\author{
Laurent Bruckler, Jean-Claude Gaudu
}

\section{To cite this version:}

Laurent Bruckler, Jean-Claude Gaudu. Utilisation des micropsychromètres pour la mesure du potentiel hydrique du sol en laboratoire et in situ. Agronomie, 1984, 4 (2), pp.171-182. hal-00884624

\section{HAL Id: hal-00884624 \\ https://hal.science/hal-00884624}

Submitted on 1 Jan 1984

HAL is a multi-disciplinary open access archive for the deposit and dissemination of scientific research documents, whether they are published or not. The documents may come from teaching and research institutions in France or abroad, or from public or private research centers.
L'archive ouverte pluridisciplinaire HAL, est destinée au dépôt et à la diffusion de documents scientifiques de niveau recherche, publiés ou non, émanant des établissements d'enseignement et de recherche français ou étrangers, des laboratoires publics ou privés. 


\title{
Utilisation des micropsychromètres pour la mesure du potentiel hydrique du sol en laboratoire et in situ
}

\author{
Laurent BRUCKLER \\ avec la collaboration technique de Jean-Claude GAUDU \\ I.N.R.A., Station de Science du Sol, Centre de Recherches d'Avignon, B.P. 91, F 84140 Montfavet.
}

RÉSUMÉ

\begin{abstract}
Après avoir rappelé le principe physique de la mesurc psychrométrique, on présente deux méthodes possibles de mesure (méthode classique et méthode du point de rosée). La prévision de la réponse psychrométrique sur la base des modèles correspondant à chaque méthode est tout à fait conforme aux courbes d'étalonnage obtenues par voie expérimentalc.

Les mesures effectuées in situ dans des conditions de forts gradients thermiques conduisent à analyser précisément les sources d'erreur liées à la non-isothermic. Sur des bases expérimentales, on conclut que la non-isothermie n'induit que des perturbations nulles ou négligeables dans les conditions cxpérimentales choisies.
\end{abstract}

Mots clés additionnels : Psychrométrie, modèles, étalonnage, mesures in situ, sources d'erreurs, isothermie, non-isothermie.

Additional key words: Psychrometric measurements, models, calibration, fields measurements, errors, isothermal conditions, non-isothermal conditions.

\section{INTRODUCTION}

L'intérêt des micropsychromètres à effet PELTIER pour la mesure du potentiel de l'eau réside, pour une grande part, dans la gamme des valeurs de potentiel accessibles avec cette technique (plusieurs dizaines de bars). Pour les végétaux, cette gamme contient l'ensemble des valeurs de potentiels foliaires ou racinaires couramment observés; pour les mesures effectuées sur le sol, elle permet de sortir des limites imposées par la tensiométrie.

Pour cette raison essentielle, la psychrométrie a suscité beaucoup de travaux, notamment depuis ceux de SPANNER (1951) qui indiquaient les possibilités, théoriques et techniques, offertes par cette méthode. Globalement, ces travaux peuvent être classés selon 3 grands axes :

A. Les recherches relatives aux bases physiques du fonctionnement des psychromètres qui devaicnt déboucher sur la modélisation de la réponse psychrométrique, c'est-à-dire de la relation entre la force électromotrice délivréc par le thermocouple et le potentiel de la phase gazeuse (RAwlins, 1966 ; PECK, 1968, 1969 ; SCOTTER, 1972).

B. La mise au point de modalités techniques de mesures en laboratoire, sur le sol ou sur les végétaux (MONTEITH \& OWEN, 1958; MillaR, 1971 $a, b$; MillaR, 1974 ; SLACK \& RIGGLE, 1980).

Beaucoup de divergences, relativement mineures, apparaissent sur les conditions de mesures jugées optimales ou sur la précision de la mesure. Mais globalement, la méthode psychrométrique elle-même n'est pas remise en cause. L'accent est plutôt mis sur la sensibilité de la réponse psychrométrique aux conditions de mesure lors de sa réalisation. 
C. L'utilisation de la mesure psychrométrique in situ, ou plus généralement en conditions non isothermes (RAWlins \& Dalton, 1967 ; MERRILl \& RaWlins, 1972 ; VALANCOGNE \& DAUDET, 1974)

Dans ce cas, les problèmes posés sont relatifs au sens de la mesure : en effet, la démonstration théorique ou expérimentale de la validité ou de la non-validité de la mesure in situ n'est pas facile, puisque les résultats acquis ne peuvent être corrélés avec ceux d'une technique indépendante et suffisamment précise sur une gamme de plusieurs dizaines de bars. De plus, les variations de température, qui peuvent a priori perturber la mesure, induisent aussi des variations réelles de l'humidité relative de la phase gazeuse, c'est-àdire de la variable à mesurer.

L'objet de ce travail est de contribuer à la clarification de ces différents points : en laboratoire, on cherchera surtout à confronter les modélisations possibles de la réponse psychrométrique aux mesures réalisées dans différentes conditions. L'analyse des données obtenues in situ cherchera à clarifier le sens de la mesure obtenue en conditions non isothermes.

\section{PRINCIPE DE LA MESURE PSYCHROMÉTRIQUE}

La mesure psychrométrique ne concerne que la phase gazeuse d'un milieu donné : de ce fait, il est abusif de parler a priori de mesure du "potentiel hydrique d'un sol" puisqu'en toute rigueur la mesure se ramène à celle d'une humidité relative ou d'une pression partielle de vapeur d'eau : il n'y a coïncidence entre les 2 termes de potentiel de l'eau du sol et d'humidité relative que dans le cas où l'équilibre entre les différentes phases est réalisé, ce qui ne constitue pas a priori le cas général, surtout en conditions non isothermes. Si l'on considère le cas idéal où l'équilibre entre les phases " solide + liquide " et gazeuse est réalisé à une température $T_{0}$, on pourra caractériser cette phase gazeuse par 3 grandeurs:

- sa pression partielle de vapeur d'eau $p$,

- son humidité relative $H_{r}=p / p_{o}\left(T_{o}\right)$,

$\mathrm{p}_{0}\left(\mathrm{~T}_{\mathrm{o}}\right)$ étant la pression de vapeur saturante à la température $\mathrm{T}_{\mathrm{s}}$,

- son potentiel hydrique $\psi$, tel que :

$$
\psi=\frac{\mathrm{RT}_{0}}{\mathrm{Mg}} \ln \left(\mathrm{p} / \mathrm{p}_{0}\right)
$$

$$
\begin{array}{ll}
\psi=\text { potentiel } & {[\mathrm{L}]} \\
\mathrm{R}=\text { constante des gaz parfaits } & {\left[\mathrm{ML}^{2} \mathrm{~T}^{-2} \text { mole }^{-1} \mathrm{~K}^{-1}\right]} \\
\mathrm{M}=\text { masse molaire de l'eau } & {\left[\mathrm{M} \mathrm{mole}^{-1}\right]} \\
\mathrm{g}=\text { accélération de la pesanteur }\left[\mathrm{L} \mathrm{T}^{-2}\right]
\end{array}
$$

Le principe cle la mesure consiste à déterminer $\left(\mathrm{p} / \mathrm{p}_{0}(\mathrm{~T})\right)$ pour la phase gazeuse située dans la chambre interne du psychromètre et supposée en équilibre avec le milieu extérieur (fig. 1). La chambre de mesure, délimitée par une capsule poreuse, renferme un thermocouple chromelconstantan ( $\mathrm{Cr} / \mathrm{Cst})$, qui comporte une jonction de «mesure " et une jonction de « référence », constituée en réalité de 2 plots de cuivre, et dont la température doit rester constante tout le long de la mesure (forte chaleur massique).

Le matériel utilisé est de type WESCOR (microvoltmètre HR 33 T).

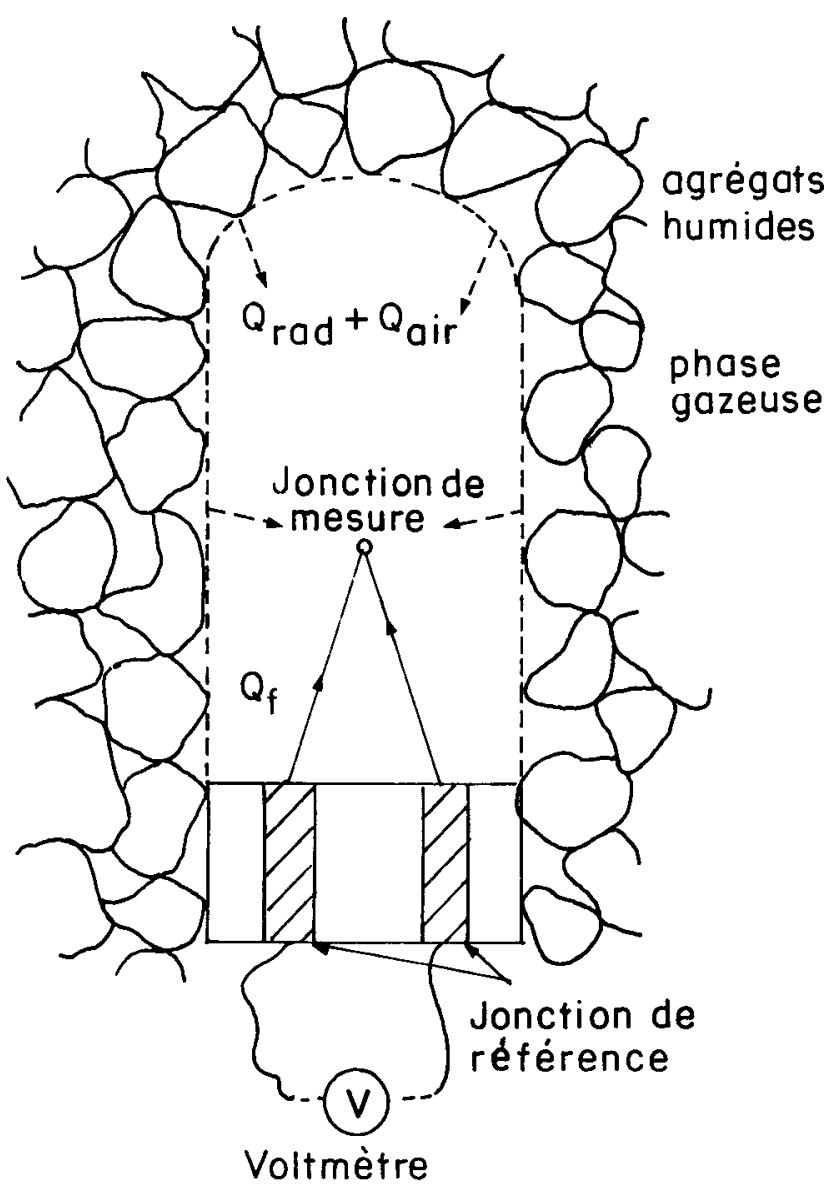

Figure 1

Représentation schématique d'un micropsychromètre (Qf, $Q_{\text {rad }}$, $Q_{\text {air }}$ : voir texte).

Schematic diagram of a micropsychrometer $\left(Q f, Q_{\text {rad }}, Q_{\text {air }}\right.$ : see text).

Dans une $1^{\text {re }}$ phase, à partir de l'état initial $\mathrm{A}$ du système, on refroidit la soudure de mesure par effet PELTIER jusqu'à condensation d'une infime quantité d'eau sur celle-ci (fig. 2, parcours $\mathrm{AB}$ ). Si le refroidissement est prolongé au-delà de la température du point de rosée $T_{r}$, la masse d'eau condensée sur la soudure de mesure augmente (fig. 2, parcours BC). A partir de l'arrêt du refroidissement, 2 méthodes de mesure sont alors possibles en utilisant le matériel cité plus haut :

- dans la méthode psychrométrique dite "classique», on laisse la soudure de mesure se réchauffer librement. Du fait de l'évaporation, la soudure de mesure prend une température d'équilibre $T_{e}$ telle que $T_{r}<T_{e}<T_{o}$ : dans ce cas, l'évaporation a lieu à une température supérieure à la température du point de rosée $T_{r}$, car cette dernière caractérise précisément un équilibre liquide-vapeur, c'est-àdire l'absence d'évaporation. On détermine enfin la relation $\mathrm{T}_{\mathrm{o}}-\mathrm{T}_{\mathrm{e}}=\mathrm{f}\left(\mathrm{p} / \mathrm{p}_{\mathrm{o}}\left(\mathrm{T}_{\mathrm{o}}\right)\right)$, par étalonnage expérimental ou modélisation.

- dans la méthode psychométrique dite « du point de rosée », après arrêt du refroidissement et réchauffement de la soudure jusqu'au point de rosée (fig. 2, parcours CB), un dispositif électronique permet de compenser par effet Peltier le réchauffement de la soudure dû aux flux radiatifs et conductifs vers la soudure de mesure : celle-ci tend alors à prendre une température d'équilibre égale à $T_{r}$. Dans ce cas, on mesure un abaissement de température $T_{o}-T_{r}=f\left(p / p_{0}\left(T_{o}\right)\right)$. 
Pression de

vapeur saturante

$p_{0}(\mathrm{~mm} \mathrm{Hg})$

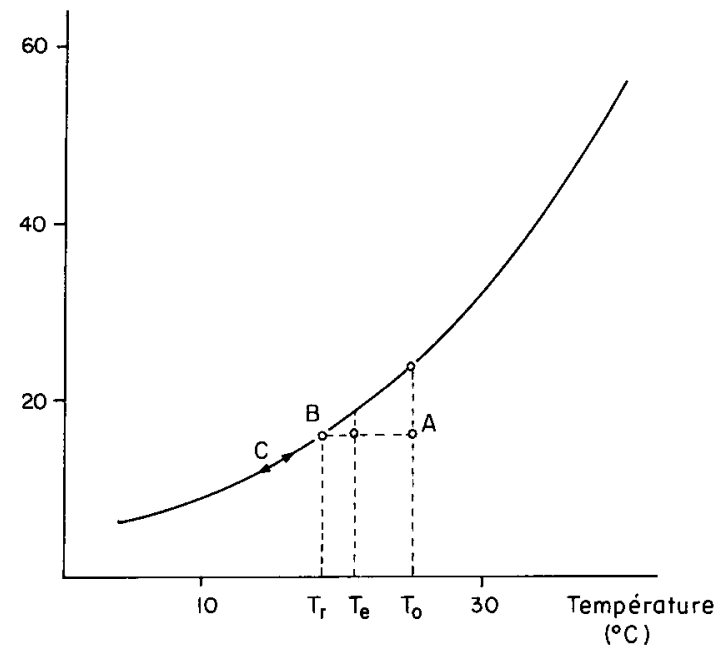

Figure 2

Pression de vapeur saturante en fonction de la température et principe de mesure des micropsychromètres.

Saturated vapor pressure versus temperature and principle of micropsychrometer use.

\section{MODÉLISATION DE LA RÉPONSE PSYCHROMÉTRIQUE}

Les 2 méthodes présentées, correspondant à des situations physiques différentes, répondent à 2 modélisations bien distinctes.

\section{A. Méthode psychrométrique classique}

Lors de la phase d'évaporation de l'eau condensée, la soudure se trouve à une température $T_{e}$, alors que le milieu environnant reste sensiblement à la température initiale $T_{o}$. Cette différence de température $\left(\mathrm{T}_{\mathrm{o}}-\mathrm{T}_{\mathrm{e}}\right)$ induit un ensemble de flux énergétiques du milieu extérieur vers la soudure.
Pour modéliser la réponse du psychromètre, RAWLINS (1966) propose d'analyser le bilan d'énergie au niveau de la soudure de la façon suivante :

\section{Hypothèses}

a) On assimile le volume interne de la chambre psychrométrique à un cylindre de rayon interne $r_{c}$ et de hauteur $2 r_{c}$. Cette hypothèse reste relativement faible puisque le constructeur fournit des capsules psychrométriques telles que $r_{c}=2,5 \mathrm{~mm}$ et de hauteur égale à 5 ou $8 \mathrm{~mm}$ selon le type choisi. Cette simplification d'ordre géométrique est modifiée dans le cas du calcul du flux de chaleur conductif dans l'air et du flux de chaleur latente, pour lesquels on assimile la soudure de mesure et la capsule psychrométrique à 2 sphères emboîtées de rayon respectifs $r_{j}$ et $r_{c}$, comptetenu du fait que $r_{j}<<r_{c}$.

b) A l'intérieur de la chambre psychrométrique, on néglige la convection par rapport à la conduction. Cette hypothèse semble acceptable si $\left(\mathrm{T}_{\mathrm{o}}-\mathrm{T}_{\mathrm{c}}\right)<1{ }^{\circ} \mathrm{C}$ (ce qui est toujours le cas) et si $\mathrm{r}_{\mathrm{c}}<4 \mathrm{~cm}$ (KYTE et al., 1953).

\section{Bilan énergétique au niveau de la soudure}

Le tableau 1 donne l'ensemble des paramètres nécessaires à l'écriture des flux énergétiques au niveau de la soudure. On distingue 3 flux du milieu extérieur vers la soudure (tabl. 2) :

- un flux de type radiatif, des parois de la chambre psychrométrique vers la jonction de mesure $\left(\mathrm{d} \mathrm{Q}_{\mathrm{rad}} / \mathrm{dt}\right)$, - un flux de type conductif à travers le volume d'air de la chambre $\left(\mathrm{d} \mathrm{Q}_{\mathrm{air}} / \mathrm{dt}\right)$,

- un flux de type conductif le long des fils du thermocouple $\left(\mathrm{d} \mathrm{Q}_{\mathrm{f}} / \mathrm{dt}\right)$.

Dans le même temps, l'évaporation de la masse d'eau condensée correspond à un flux de chaleur latente (d Q $\mathrm{evap} / \mathrm{dt}$ ).

L'écriture du bilan énergétique au niveau de la soudure s'écrit alors :

$$
\frac{\mathrm{dQ}_{\mathrm{rad}}}{\mathrm{dt}}+\frac{\mathrm{dQ}_{\mathrm{air}}}{\mathrm{dt}}+\frac{\mathrm{dQ}_{\mathrm{f}}}{\mathrm{dt}}=\frac{\mathrm{dQ}_{\mathrm{evap}}}{\mathrm{dt}} .
$$

\section{TABLEAU 1}

Paramètres intervenant dans le modèle relatif à la méthode psychrométrique classique. Parameters used in the model for the psychrometric method.

\begin{tabular}{|c|c|c|c|}
\hline $\begin{array}{l}\text { Paramètres géométriques } \\
\text { du psychromètre }\end{array}$ & $\begin{array}{l}\text { Paramètres physiques } \\
\text { du thermocouple }\end{array}$ & $\begin{array}{c}\text { Paramètres physiques } \\
\text { du milieu }\end{array}$ & $\begin{array}{l}\text { Fonctions physiques } \\
\text { élémentaires }\end{array}$ \\
\hline $\begin{array}{l}\text { Rayon de la jonction } \\
\text { de mesure }\left(r_{j}\right) \\
{[\mathrm{L}]}\end{array}$ & $\begin{array}{l}\text { Conductivité thermique } \\
\text { des fils du thermocouple }\left(\mathrm{k}_{\mathrm{f}}\right) \\
{\left[\mathrm{MLT}^{-3} \mathrm{~K}^{-1}\right]}\end{array}$ & $\begin{array}{l}\text { Température du milieu } \\
\qquad\left(\mathrm{T}_{\mathrm{o}}\right)\end{array}$ & $\begin{array}{c}\text { Pression de vapeur saturante } \\
\left(\mathrm{p}_{\mathrm{o}}(\mathrm{T})\right) \\
{\left[\mathrm{ML}^{-1} \mathbf{T}^{-2}\right]}\end{array}$ \\
\hline $\begin{array}{c}\text { Rayon de la chambre } \\
\text { psychrométrique }\left(r_{c}\right) \\
{[L]}\end{array}$ & $\begin{array}{l}\text { Coefficient Peltier } \\
\text { du thermocouple }(\alpha) \\
{\left[\mathrm{ML}^{2} \mathbf{Q}^{-1} \mathrm{~T}^{-2} \mathrm{~K}^{-1}\right]}\end{array}$ & $\begin{array}{c}\text { Coefficient de diffusion } \\
\text { de la vapeur d'eau dans l'air (D) } \\
{\left[\mathbf{L}^{2} \mathbf{T}^{-1}\right]}\end{array}$ & $\begin{array}{c}\text { Pente de la courbe de pression } \\
\text { de vapeur saturante }\left(\mathrm{p}_{\mathrm{o}}^{\prime}(\mathrm{T})\right) \\
{\left[\mathrm{ML}^{-1} \mathrm{~T}^{-2} \mathrm{~K}^{-1}\right]}\end{array}$ \\
\hline \multirow[t]{2}{*}{$\begin{array}{l}\text { Rayon du fil du thermocouple }\left(r_{f}\right) \\
{[L]}\end{array}$} & & $\begin{array}{l}\text { Conductivité thermique de l'air }\left(\mathrm{k}_{\mathrm{a}}\right) \\
{\left[\mathrm{MLT}^{-3} \mathrm{~K}^{-1}\right]}\end{array}$ & $\begin{array}{c}\text { Chaleur latente } \\
\text { de vaporisation }(\mathrm{L}) \\
{\left[\mathrm{L}^{2} \mathrm{~T}^{-2}\right]}\end{array}$ \\
\hline & & $\begin{array}{c}\text { Potentiel hydrique } \\
\text { de la phase gazeuse }(\psi) \\
{[\mathrm{L}]}\end{array}$ & \\
\hline
\end{tabular}


TABLEAU 2

Bilan énergétique au niveau de la soudure de mesure et force électromotrice résultante (méthode psychrométrique classique). Energy balance at the measurement junction and predicted e.m.f. (psychrometric method).

\begin{tabular}{|c|c|c|}
\hline Grandeur physique & Expression algébrique & Dimension \\
\hline Flux radiatif & $\frac{\mathrm{d} \mathrm{Q}_{\mathrm{rad}}}{\mathrm{dt}}=16 \pi \sigma \mathrm{r}_{\mathrm{j}}^{2} \mathrm{~T}_{0}^{3}\left(\mathrm{~T}_{\mathrm{o}}-\mathrm{T}_{\mathrm{c}}\right)$ & {$\left[\mathrm{ML}^{2} \mathrm{~T}^{-3}\right]$} \\
\hline Flux conductif à travers l'air & $\frac{d \text { Qair }}{d t}=\frac{4 \pi r_{j} r_{c} k_{a}}{r_{c}-r_{j}}\left(T_{o}-T_{e}\right)$ & {$\left[\mathrm{ML}^{2} \mathrm{~T}^{-3}\right]$} \\
\hline $\begin{array}{l}\text { Flux conductif le long des fils du } \\
\text { thermocouple }\end{array}$ & $\frac{\mathrm{d} Q_{f}}{d t}=\pi r_{f}^{2} k_{f} \cdot \gamma \cdot\left(T_{o}-T_{c}\right)$ & {$\left[\mathrm{ML}^{2} \mathrm{~T}^{-3}\right]$} \\
\hline Flux de chaleur latente & $\frac{d Q_{\text {cvap }}}{d t}=\frac{4 \pi r_{j} r_{c}}{r_{c}-r_{j}} \cdot L D \frac{M}{R T_{o}}\left\{p_{o}\left(T_{o}\right)\left[1-c\left(\frac{M g}{R T_{o}} \cdot \psi\right)\right]-\left(T_{o}-T_{e}\right) p_{o}^{\prime}\left(T_{o}\right)\right\}$ & {$\left[\mathrm{ML}^{2} \mathrm{~T}^{-3}\right]$} \\
\hline Force électromotrice résultante & $\mathrm{U}=\frac{\left[\left(4 \mathrm{r}_{\mathrm{j}} \mathrm{r}_{\mathrm{c}}\right) /\left(\mathrm{r}_{\mathrm{c}}-\mathrm{r}_{\mathrm{j}}\right)\right] \alpha . \mathrm{D} \cdot \mathrm{L}\left(\mathrm{M} / \mathrm{RT}_{\mathrm{o}}\right) \mathrm{p}_{\mathrm{o}}\left(\mathrm{T}_{\mathrm{o}}\right)\left[1-\mathrm{e}\left(\frac{\mathrm{Mg}}{\mathrm{RT}} \cdot \psi\right)\right]}{16 \sigma \mathrm{r}_{\mathrm{j}}^{2} \mathrm{~T}_{\mathrm{o}}^{3}+\mathrm{r}_{\mathrm{f}}^{2} \mathrm{k}_{\mathrm{f}} \gamma+\left[\left(4 \mathrm{r}_{\mathrm{c}} \mathrm{r}_{\mathrm{j}}\right) /\left(\mathrm{r}_{\mathrm{c}}-\mathrm{r}_{\mathrm{j}}\right)\right]\left[\mathrm{k}_{\mathrm{a}}+\mathrm{D} \cdot \mathrm{L}\left(\mathrm{M} / \mathrm{RT} \mathrm{T}_{\mathrm{o}}\right) \mathrm{p}_{\mathrm{o}}^{\prime}\left(\mathrm{T}_{\mathrm{o}}\right)\right]}$ & {$\left[\mathrm{ML}^{2} \mathrm{~T}^{-2} \mathrm{Q}^{-1}\right]$} \\
\hline $\begin{array}{l}\text { (a) } \sigma: \text { Constante de Stephan Bol } \\
\text { (b) } \gamma=2 \cdot\left\{\frac{\left(2 \pi \mathrm{k}_{\mathrm{a}}\right) /\left[\ln \left(\mathrm{r}_{\mathrm{c}} / \mathrm{r}_{\mathrm{f}}\right)\right]}{\pi \mathrm{r}_{\mathrm{f}}^{2} \mathrm{k}}\right.\end{array}$ & $\begin{array}{l}\text { ltzmann }\left[\mathrm{MT}^{-3} \mathrm{~K}^{-4}\right] \\
+8 \pi \mathrm{r}_{\mathrm{f}} \sigma \mathrm{T}_{\mathrm{o}}^{3} \\
\begin{array}{ll}\mathrm{f}_{\mathrm{f}} \\
\}^{1 / 2}\end{array}\end{array}$ & \\
\hline
\end{tabular}

De (2), on déduit alors l'expression de l'abaissement de température $\left(\mathrm{T}_{0}-\mathrm{T}_{\mathrm{e}}\right)$ ou de la force électromotrice correspondante $\mathrm{U}$ aux bornes du thermocouple correspondant à un potentiel $\psi$ de la phase gazeuse (tabl. 2).

\section{B. Méthode du point de rosée.}

Contrairement au cas précédent, la stabilisation de la température au point de rosée implique qu'il y a équilibre strict « liquide-vapeur » et qu'il n'y a ni vaporisation, ni condensation: l'apport énergétique du milieu extérieur se trouve ainsi totalement compensé par l'extraction de chaleur du thermocouple par effet PELTIER. La modélisation se ramène dans ce cas à l'obtention d'une relation physique entre l'abaissement de température $\left(T_{0}-T_{r}\right)$ et le potentiel de l'eau $\psi$ correspondant à une pression partielle réelle de la vapeur d'eau $p$ :

On a ainsi :

$$
\mathrm{p}=\mathrm{p}_{\mathrm{o}}\left(\mathrm{T}_{\mathrm{r}}\right)
$$

et d'après la loi de Kelvin :

$$
\mathrm{p}=\mathrm{p}_{0}\left(\mathrm{~T}_{0}\right) \exp \left(\frac{\mathrm{Mg}}{\mathrm{RT}_{0}} \cdot \psi\right)
$$

La combinaison de (3), (4) permet d'écrire :

$$
\mathrm{p}_{0}\left(\mathrm{~T}_{\mathrm{r}}\right)=\mathrm{p}_{0}\left(\mathrm{~T}_{0}\right) \exp \left(\frac{\mathrm{Mg}}{\mathrm{RT}_{0}} \cdot \psi\right)
$$

soit encore :

$$
\mathrm{p}_{0}\left(\mathrm{~T}_{\mathrm{r}}\right)-\mathrm{p}_{0}\left(\mathrm{~T}_{0}\right)+\mathrm{p}_{0}\left(\mathrm{~T}_{0}\right)=\mathrm{p}_{0}\left(\mathrm{~T}_{0}\right) \exp \left(\frac{\mathrm{Mg}}{\mathrm{RT}_{0}} \cdot \psi\right)
$$

En approchant la pente de la courbe de pression de vapeur saturante $\mathrm{p}_{\mathrm{o}}^{\prime}\left(\mathrm{T}_{\mathrm{o}}\right) \mathrm{par}$ :

$$
\mathrm{p}_{0}^{\prime}\left(\mathrm{T}_{0}\right)=\frac{\mathrm{p}_{0}\left(\mathrm{~T}_{0}\right)-\mathrm{p}_{0}\left(\mathrm{~T}_{\mathrm{r}}\right)}{\mathrm{T}_{0}-\mathrm{T}_{\mathrm{r}}}
$$

on aura finalement :

$$
\left(\mathrm{T}_{0}-\mathrm{T}_{\mathrm{r}}\right)=\frac{\mathrm{p}_{0}\left(\mathrm{~T}_{0}\right)}{\mathrm{p}_{0}^{\prime}\left(\mathrm{T}_{0}\right)} \cdot\left[1-\exp \left(\frac{\mathrm{Mg}}{\mathrm{RT}_{0}} \cdot \psi\right)\right] .
$$

L'équation (8) peut s'exprimer également par rapport à la force électromotrice lue $U$ :

$$
\mathrm{U}=\alpha \frac{\mathrm{p}_{0}\left(\mathrm{~T}_{0}\right)}{\mathrm{p}_{0}\left(\mathrm{~T}_{0}\right)} \cdot\left[1-\exp \left(\frac{\mathrm{Mg}}{\mathrm{RT}_{0}} \cdot \psi\right)\right] .
$$

Enfin, en utilisant la relation de Clausius-Clapeyron

$$
\mathrm{p}_{0}^{\prime}\left(\mathrm{T}_{0}\right)=\mathrm{p}_{0}\left(\mathrm{~T}_{0}\right) \cdot \frac{\mathrm{L}}{\mathrm{RT}_{0}^{2}} \text {. }
$$

On pourra aussi écrire :

$$
\mathrm{U}=\alpha \frac{\mathrm{RT}_{0}^{2}}{\mathrm{~L}}\left[1-\exp \left(\frac{\mathrm{Mg}}{\mathrm{RT}_{0}} \cdot \psi\right)\right]
$$

\section{Comparaison des deux méthodes}

Le tableau 3 donne la valeur des paramètres, fonctions de la température, qui ont été utilisés pour les calculs des relations $\mathrm{U}=\mathrm{f}(\psi)$.

Les caractéristiques géométriques des psychromètres sont obtenues auprès du constructeur, tandis que la conductivité 
TABLEAU 3

Paramètres physiques en fonction de la température (méthode psychrométrique classique). Physical parameters versus temperature (psychrometric method).

\begin{tabular}{|c|c|c|c|c|c|c|}
\hline $\begin{array}{c}\mathrm{T} \\
\left({ }^{\circ} \mathrm{C}\right)\end{array}$ & $\begin{array}{c}\mathrm{po}_{0}(\mathrm{~T}) \\
(\mathrm{Pa})\end{array}$ & $\begin{array}{c}\mathrm{p}_{\mathrm{o}}^{\prime}(\mathrm{T}) \\
\left(\mathrm{Pa}^{\circ}{ }^{\circ} \mathrm{C}\right)\end{array}$ & $\begin{array}{c}\mathrm{L} \\
(\mathrm{J} / \mathrm{kg}) \\
\left(\mathrm{x} \quad 10^{-6}\right)\end{array}$ & 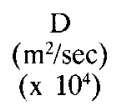 & $\begin{array}{c}k_{a} \\
\left(W / m^{\circ} \mathrm{C}\right) \\
\left(x \quad 10^{2}\right)\end{array}$ & 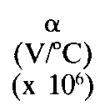 \\
\hline 5 & 879,2 & 58,1 & 2,478 & 0,226 & 2,41 & 58,5 \\
\hline 10 & 1216,3 & 77,1 & 2,467 & 0,233 & 2,44 & 59,0 \\
\hline 15 & 1775,9 & 101,8 & 2,455 & 0,240 & 2,48 & 59,5 \\
\hline 20 & 2288,5 & 133,8 & 2,444 & 0,248 & 2,51 & 60,0 \\
\hline 25 & 3114,9 & 175,1 & 2,433 & 0,255 & 2,55 & 60,5 \\
\hline 30 & 4218,2 & 228,0 & 2,422 & 0,263 & 2,58 & 61,0 \\
\hline 35 & 5681,9 & 295,5 & 2,412 & 0,270 & 2,61 & 61,5 \\
\hline 40 & 7618,9 & 381,7 & 2,401 & 0,278 & 2,65 & 62,0 \\
\hline
\end{tabular}

thermique du thermocouple $(\mathrm{Cr} / \mathrm{Cst})$ est celle donnéc par RAWLINS (1966) :

$$
\begin{aligned}
& \mathbf{r}_{\mathrm{c}}=0,250 \mathrm{~cm} \\
& \mathbf{r}_{\mathrm{j}}=0,625 \cdot 10^{-2} \mathrm{~cm} \\
& \mathbf{r}_{\mathrm{f}}=0,127 \cdot 10^{-2} \mathrm{~cm} \\
& \mathrm{k}_{\mathrm{f}}=10,03 \mathrm{~W} / \mathrm{m}^{\circ} \mathrm{C}
\end{aligned}
$$

Les principales caractéristiques des 2 méthodes et des modèles correspondants sont les suivantes:

1. La réponse psychrométrique $\mathrm{U}$ ou $\left(\mathrm{T}_{\mathrm{o}}-\mathrm{T}_{\mathrm{e}}\right)$ caractérisant la $1^{\text {re }}$ méthode dépend des caractéristiques géométriques et physiques du psychromètre. Si la technique de construction des psychromètres aboutit à de très faibles fluctuations entre psychromètres des termes $\mathbf{r}_{\mathrm{j}}$ et $\mathbf{r}_{\mathrm{f}}$ notamment, on peut s'attendre à ce que les réponses des différents psychromètres soient pratiquement assimilables à un ćtalonnage unique. Par contre, une modification des termes $r_{j}$ ou $r_{f}$ importante entre 2 psychromètres, de constructeurs différents par exemple, aboutit à une modification non négligeable de la réponse psychrométrique. Le tableau 4 et la figure 3 illustrent la sensibilité de la réponse du psychromètre aux paramètres géométriques de ce dernier.

2. L'abaissement de température $\left(T_{\mathrm{o}}-\mathrm{T}_{\mathrm{r}}\right)$ étant supérieur à $\left(\mathrm{T}_{\mathrm{o}}-\mathrm{T}_{\mathrm{c}}\right)$, la méthode du point de rosée fournit nécessairement des forces électromotrices $U$ plus élevées: cette dernière méthode est donc la plus sensible, ce que précisent les calculs du tableau 5.

3. Les 2 méthodes de mesures sont sensibles aux variations de température du milieu, soit directement $\left(T_{0}\right)$, soit indirectement, par l'intermédiaire des paramètres dépendant de $\mathrm{T}_{\mathrm{o}}$ (tabl. 3). Après calculs, il s'avère cependant que la méthode psychrométrique classique est beaucoup plus sensible que la méthode du point de rosée (fig. 4): les exigences relatives à la connaissance de la température réelle du milieu sont donc beaucoup plus importantes pour la $1^{\text {re }}$ méthode.

Sur l'ensemble de ces 3 points, la méthode du point de rosée apparaît nettement plus performante que la méthode classique. Elle nécessite cependant 2 mesures préliminaires :

- comme pour la méthode classique, la mesure de température du milieu (en fait, la température du psychromètre). Cette détermination s'effectue grâce à un thermocouple supplémentaire incorporé au psychromètre. La température mesurée à l'aide de cette jonction ne présente pratiquement pas de biais par rapport à la température mesurée avec un thermomètre de précision et ne nécessite donc pas de correction pour les mesures courantes $\left(0,3{ }^{\circ} \mathrm{C}\right.$ d'écart maximal pour la gamme 5 à $50^{\circ} \mathrm{C}$ ) ;

\section{TABLEAU 4}

Effet des variations des paramètres géométriques du psychromètre sur la force électromotrice résultante (méthode psychrométrique

\begin{tabular}{|c|c|c|}
\hline $\begin{array}{l}\text { Paramètre } \\
\text { géométrique }\end{array}$ & $\begin{array}{c}(\stackrel{1}{)}) \\
\text { Valeur } \\
\text { numérique }(\mathrm{cm})\end{array}$ & $\begin{array}{c}\text { Sensibilité } \\
\text { de la mesure } \\
\text { à } 20^{\circ} \mathrm{C} \\
(\mu \mathrm{V} / \mathrm{bar})\end{array}$ \\
\hline$r_{c}$ & $\begin{array}{l}0,225 \\
0,250 \\
0,275\end{array}$ & $\begin{array}{l}0,349 \\
0,350 \\
0,350\end{array}$ \\
\hline$r_{j}$ & $\begin{array}{l}0,563 \cdot 10^{-2} \\
0,625 \cdot 10^{-2} \\
0,688 \cdot 10^{-2}\end{array}$ & $\begin{array}{l}0,333 \\
0,350 \\
0,361\end{array}$ \\
\hline $\mathbf{r}_{f}$ & $\begin{array}{l}0,114 \cdot 10^{-2} \\
0,127 \cdot 10^{-2} \\
0,140 \cdot 10^{-2}\end{array}$ & $\begin{array}{l}0,366 \\
0,350 \\
0,340\end{array}$ \\
\hline
\end{tabular}
classique).

Effect of variation in the geometrical parameters of the psychrometer on the e.m.f. (psychrometric method).

(1) Les valeurs centrales sont données par le constructeur. Les valeurs extrêmes correspondent à une variation de \pm 10 p. 100 autour des valeurs centrales.

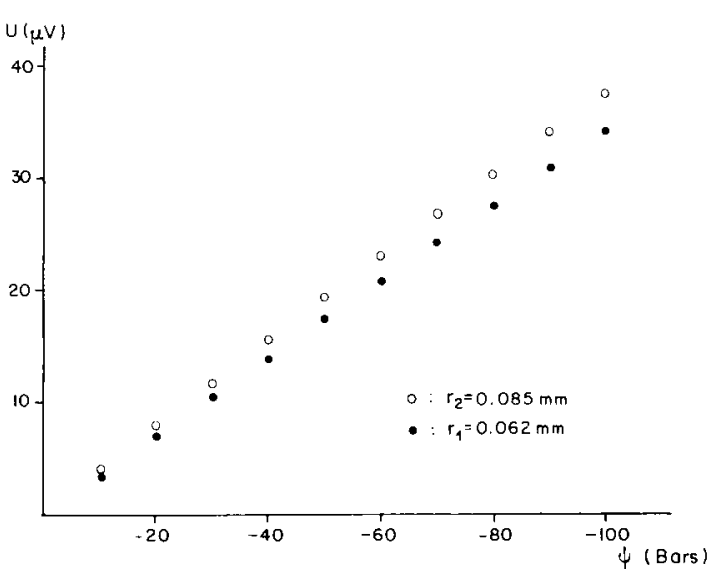

Figure 3

f.e.m. calculée en fonction du potentiel de l'eau pour 2 rayons de la soudure de mesure (méthode psychrométrique classique).

Calculated e.m.f. versus water potential for two radii of the measurement junction (psychrometric method). 
TABLEAU 5

Abaissements de température et f.e.m. calculées pour deux méthodes de mesures psychrométriques $\left(20^{\circ} \mathrm{C}\right)$.

Calculated temperature depression and e.m.f. according to the psychrometric method $\left({ }^{1}\right)$ or dew-point method $\left({ }^{2}\right)$ at $20^{\circ} \mathrm{C}$.

\begin{tabular}{|c|c|c|c|c|c|}
\hline $\begin{array}{l}\text { Potentiel } \\
\text { (bar) }\end{array}$ & $\begin{array}{l}\text { Humidité } \\
\text { relative }\end{array}$ & $\begin{array}{c}\Delta \mathrm{T}\left({ }^{1}\right) \\
\left({ }^{\circ} \mathrm{C}\right)\end{array}$ & $\begin{array}{c}\Delta \mathrm{T}\left({ }^{2}\right) \\
\left({ }^{\circ} \mathrm{C}\right)\end{array}$ & $\begin{array}{l}U\left({ }^{1}\right) \\
(\mu V)\end{array}$ & $\begin{array}{l}U\left(^{2}\right) \\
(\mu V)\end{array}$ \\
\hline 0 & 1,0000 & 0,0 & 0,0 & 0,0 & 0,0 \\
\hline-5 & 0,9926 & 0,029 & 0,060 & 1,8 & 3,6 \\
\hline-10 & 0,9926 & 0,058 & 0,119 & 3,5 & 7,2 \\
\hline-15 & 0,9830 & 0,087 & 0,179 & 5,2 & 10,7 \\
\hline-20 & 0,9853 & 0,116 & 0,238 & 7,0 & 14,3 \\
\hline-25 & 0,9817 & 0,145 & 0,297 & 8,7 & 17,8 \\
\hline-30 & 0,9781 & 0,174 & 0,355 & 10,4 & 21,3 \\
\hline-35 & 0,9745 & 0,202 & 0,414 & 12,1 & 24,8 \\
\hline-40 & 0,9709 & 0,231 & 0,472 & 13,8 & 28,3 \\
\hline-45 & 0,9673 & 0,259 & 0,530 & 15,6 & 31,8 \\
\hline-50 & 0,9637 & 0,287 & 0,588 & 17,2 & 35,3 \\
\hline-55 & 0,9602 & 0,316 & 0,646 & 18,9 & 38,7 \\
\hline-60 & 0,9566 & 0,344 & 0,703 & 20,6 & 42,2 \\
\hline-65 & 0,9531 & 0,372 & 0,760 & 22,3 & 45,6 \\
\hline-70 & 0,9496 & 0,400 & 0,817 & 24,0 & 49,0 \\
\hline-80 & 0,9426 & 0,455 & 0,930 & 27,3 & 55,8 \\
\hline-90 & 0,9356 & 0,510 & 1,043 & 30,6 & 62,6 \\
\hline-100 & 0,9287 & 0,564 & 1,155 & 33,9 & 69,3 \\
\hline
\end{tabular}

$\Delta \mathrm{T}$ : Abaissement de température.

(') Méthode psychrométrique classique.

$\left({ }^{2}\right)$ Méthode du point de rosée.

Sensibilité en $\mu \mathrm{V} / \mathrm{bar}:\left({ }^{1}\right): 0,35 ;\left({ }^{2}\right): 0,72$.

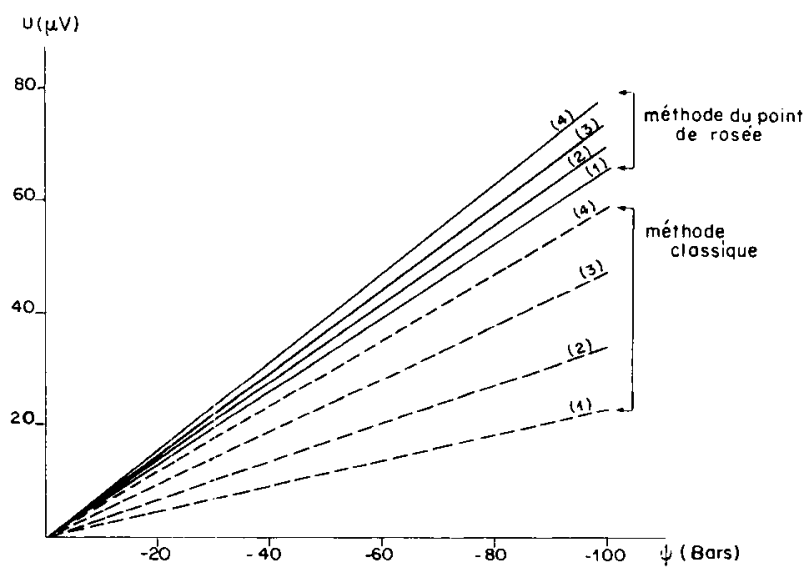

Figure 4

f.e.m. calculée en fonction du potentiel de l'eau pour deux méthodes de mesures (méthode classique et point de rosée).

Predicted e.m.f. versus water potential for two measurement methods (psychrometric and dew point method).

(1): $10^{\circ} \mathrm{C} ;(2): 20^{\circ} \mathrm{C} ;(3): 30^{\circ} \mathrm{C} ;(4): 40^{\circ} \mathrm{C}$

- la mesure préalable, pour chaque psychromètre, de la force électromotrice maximale $\mathrm{U}_{\max }$ délivrée par le thermocouple de mesure lorsqu'il est refroidi en atmosphère sèche par un courant d'une intensité donnée $(\sim 8 \mathrm{~mA})$. On peut montrer que cette valeur $\mathrm{U}_{\max }$, analogue à la grandeur $\pi_{\mathrm{v}}$ définie par le constructeur, est une fonction de la température : ceci est confirmé par l'expérience (fig. 5), puisque $\mathrm{U}_{\max }$ est une fonction linéaire de la température $\left(\mathrm{r}^{2}>0,99\right)$, variable d'un psychromètre à l'autre.

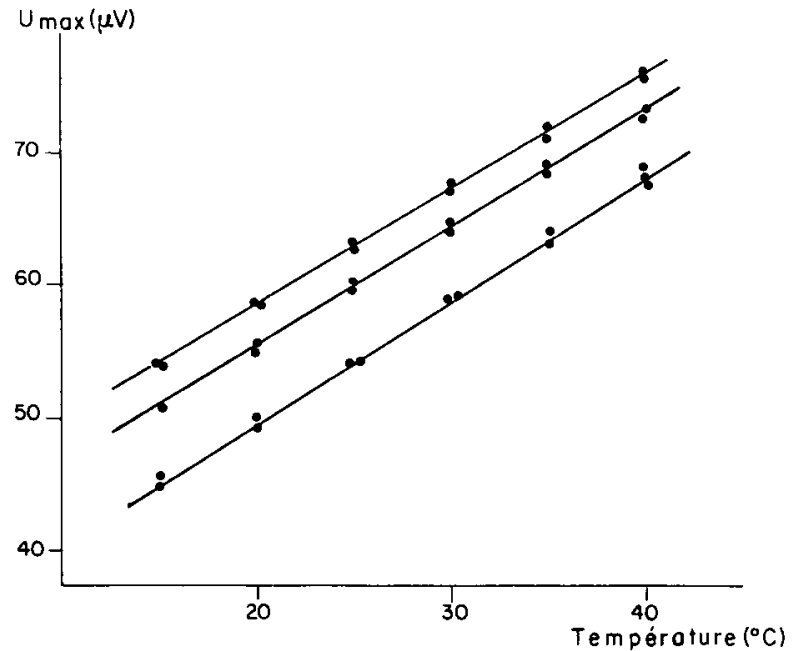

Figure 5

f.e.m. maximale en atmosphère sèche en fonction de la température (3 psychromètres).

Maximal e.m.f. with dry water vapor versus temperature (3 psychrometers).

\section{Remarques :}

1) PECK (1968) a développé un modèle proche de celui de RAWLINS (1966). En fait, les calculs montrent que les résultats numériques obtenus sont quasiment identiques pour les 2 modèles. Nous ne conserverons donc que le modèle de RAwLINS (1966).

2) Les relations calculées de la forme $U=f(\psi)$ ne sont pas rigoureusement linéaires. Avec une très faible erreur cependant, elles sont pratiquement assimilables à des demidroites.

3) Le potentiel $\psi$ donné par la relation (1) est de dimension [L]. Dans la suite, nous exprimerons cependant $\psi$ en bar $\left[\mathrm{ML}^{-1} \mathrm{~T}^{-2}\right]$, compte-tenu du caractère général de cette unité utilisée en psychrométric.

\section{IV. ÉTALONNAGE DES PSYCHROMÈTRES : VALI- DATION DES MODĖLES ET PROBLÈMES POSÉS}

L'étalonnage s'effectue par mise en équilibre de la chambre psychrométrique avec une phase gazeuse à potentiel connu, fixé par une solution de $\mathrm{NaCl}$ (LANG, 1967). La température est contrôlée au $1 / 100^{\mathrm{e}}$ de degré, au moins dans le cas où le bain thermostaté est à $20^{\circ} \mathrm{C}$, température d'ambiance de la pièce de travail. Deux techniques d'étalonnage sont utilisées :

- dans le cas des psychromètres à céramique (WESCOR, PCT 55-15) protégés par une capsule dont le système poral est fin, on plonge directement le psychromètre dans la solution. Cette technique a l'avantage de placer la chambre de mesure dans des conditions thermiques relativement stables.

- dans le cas des psychromètres à grille, protégés par une simple trame métallique (WESCOR, PST 55-15), la chambre est nécessairement placée en phase gazeuse au-dessus de la solution. Les mesures sont effectuées après un temps d'équilibre donné par la figure 6 .

A cet égard, le problème du temps d'équilibre, largement développé dans la littérature, doit d'ailleurs être abordé avec prudence : en effet, un temps d'équilibre observé est toujours très contingent des conditions réelles de mesures. 


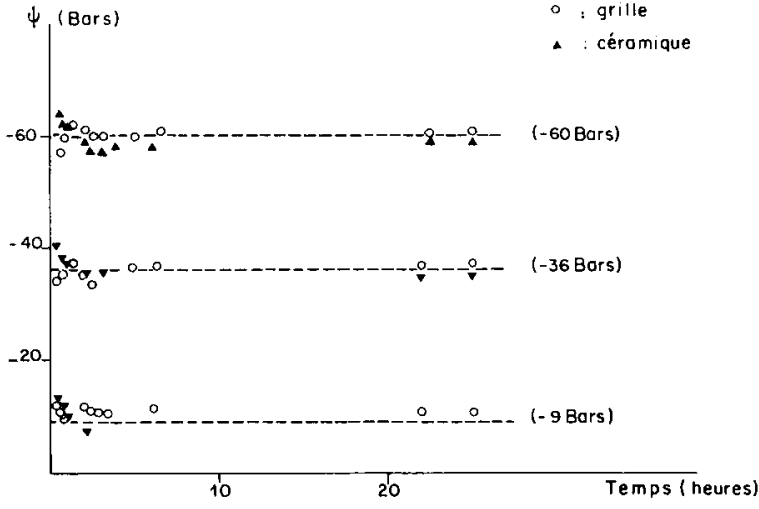

Figure 6

Potentiel hydrique mesuré en fonction du temps pour 3 valeurs du potentiel en phase gazeuse.

Measured water potential versus time for three water potential values in vapor phase.

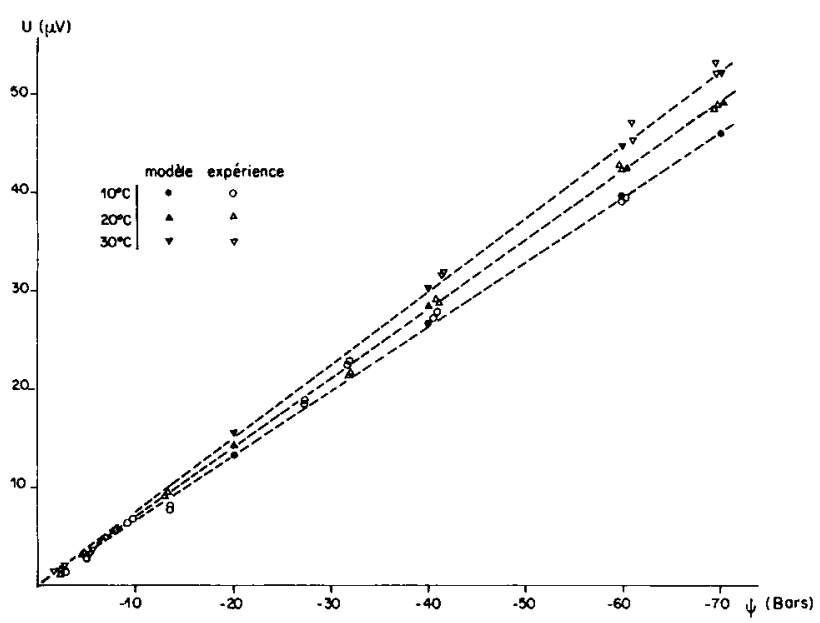

Figure 8

Courbes d'étalonnage à 3 températures (point de rosée).

Calibration curves at 3 temperatures (dew point method).

Pour un même potentiel osmotique, les temps d'équilibre peuvent être très différents selon que l'atmosphère interne du psychromètre avant mise en contact est « sèche» ou « humide». De plus, toutes les courbes de « temps d'équilibre » obtenues en laboratoire sont d'un intérêt très limité pour la mesure in situ : Dans ce cas, on n'a jamais affaire à un déséquilibre brutal entre l'atmosphère initiale de la chambre du psychromètre et le milieu, mais à une succession continue d'états d'équilibres infiniment proches les uns des autres.

La comparaison entre la prévision des relations $U=f(\psi)$ et l'étalonnage expérimental selon les 2 méthodes donne des résultats très satisfaisants (fig. $7,20^{\circ} \mathrm{C}$, psychromètres à céramique).

Il apparaît ainsi :

- que, dans la méthode classique, les hypothèses de calcul proposées par RAWLINS semblent tout à fait adéquates et que les psychromètres ont des caractéristiques géométriques très proches les unes des autres ;

- que, dans la méthode du point de rosée, la convergence vers la température $T_{r}$ est correctement assurée. Cette conclusion reste d'ailleurs valide lorsqu'on élargit la gamme des températures d'étalonnage testées (fig. 8, psychromètres à céramique).

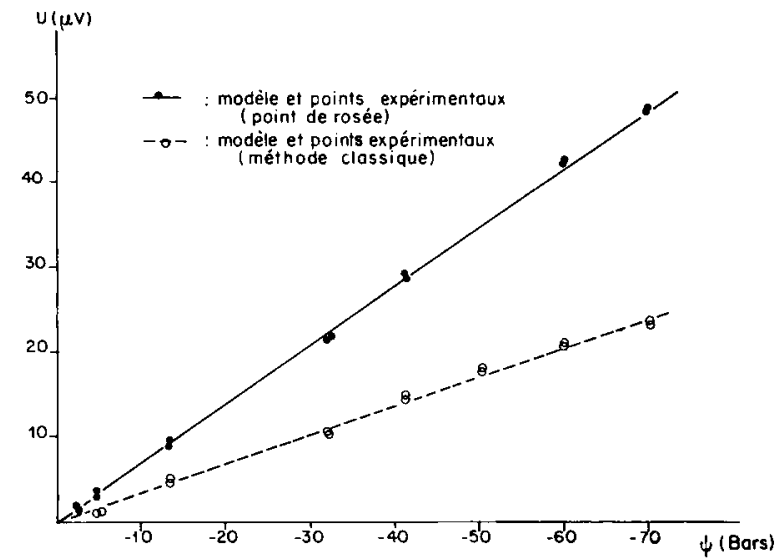

Figure 7

Courbes d'étalonnage théorique et expérimentale.

Predicted and experimental calibration curves.

Dans le cas des psychromètres à grille placés en phase gazeuse, certains étalonnages sont totalement aberrants. Un cas typique est donné par la figure 9 : l'étalonnage à $10^{\circ} \mathrm{C}$ présente, outre une forte variabilité, une déviation systématique par rapport à la prévision ou à l'étalonnage expérimental fourni par les psychromètres à céramique. Si une partie de la variabilité peut être liée à l'instabilité thermique du volume gazeux, le placement des points expérimentaux au-dessus de la courbe prévisionnelle s'interprète comme un effet purement thermique : le bac, théoriquement à $10^{\circ} \mathrm{C}$, étant placé dans une pièce à $20^{\circ} \mathrm{C}$, les gradients thermiques induits entraînent une tendance systématique au réchauffement qui correspond à une diminution apparente de l'humidité relative (fig. 2).

\section{MESURES PSYCHROMÉTRIQUES IN SITU}

\section{A. Conditions de mesures}

Les psychromètres à céramique sont placés horizontalement, sous sol nu en voie de desséchement intense de $-1 \mathrm{~cm}$ à $-25 \mathrm{~cm}$, selon les cotes suivantes: $-1 \mathrm{~cm} ;-1,5 \mathrm{~cm}$; $-2 \mathrm{~cm} ;-2,5 \mathrm{~cm} ;-3 \mathrm{~cm} ;-3,5 \mathrm{~cm} ;-4 \mathrm{~cm} ;-4,5 \mathrm{~cm}$; $-5 \mathrm{~cm} ;-7 \mathrm{~cm} ;-10 \mathrm{~cm} ;-25 \mathrm{~cm}$. La position horizontale permet de limiter le moins possible les échanges gazeux verticaux (VALANCOGNE \& DAUDET 1974).

Les mesures sont effectuées plusieurs fois par jour selon les 2 méthodes, dans des conditions où les amplitudes thermiques peuvent être particulièrement élevées (en surface, cette amplitude peut dépasser $30^{\circ} \mathrm{C}$ au cours d'une journée).

\section{B. Analyse globale des résultats}

La figure 10 présente l'évolution du potentiel mesuré en fonction du temps à différentes profondeurs (méthode du point de rosée). Par souci de clarté, seules quelques profondeurs sont indiquées, mais toutes les autres - sauf une - s'intercalent sur cette figure. L'analyse qualitative de ces résultats permet de dégager les points suivants :

1) Quelle que soit la profondeur, les mesures présentent des oscillations régulières en fonction de l'heure du jour. Ces oscillations, qui s'amortissent avec la profondeur, sont corrélées avec les variations thermiques. Elles sont cepen- 


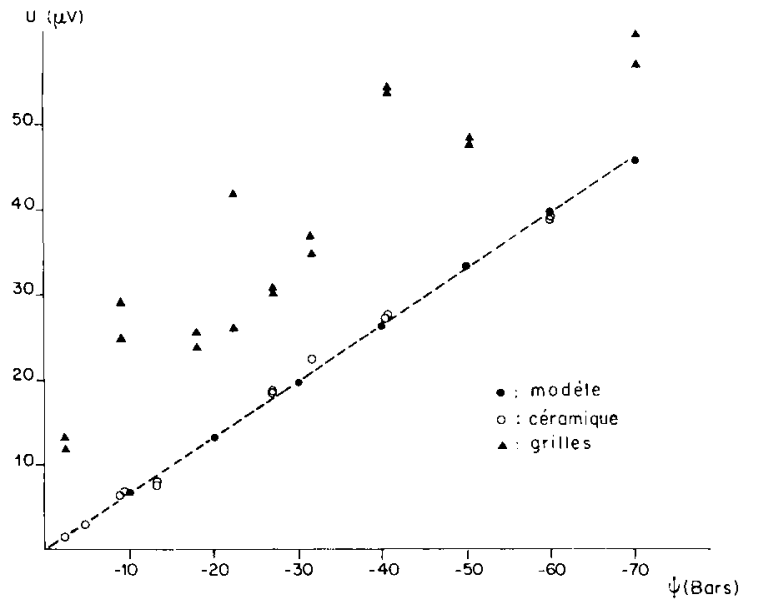

Figure 9

Courbes d'étalonnage pour des psychromètres placés en phase liquide (céramique) ou en phase gazeuse (grille).

Calibration curves for psychrometers in liquid phase (ceramic) or vapor phase (stee!).

dant corrélées également avec l'humidité relative de l'air qui varie dans le même temps. A partir de cette double liaison globale, il est impossible de conclure sur la validité ou la non-validité de la mesure psychrométrique in situ.

En fait, unc observation plus détaillée des courbes d'évolution de la température et du potentiel en fonction de l'heure du jour (fig. 11) montre que ces 2 courbes ne sont ni strictement superposables, ni simplement translatées: les maximums des 2 fonctions ne coïncident pas et les vitesses de décroissance sont différentes. Ainsi (fig. 11), une même valeur $\psi=-5$ bars peut être obtenue le matin $\left(24,5^{\circ} \mathrm{C}\right)$ ou l'après-midi $\left(37.1^{\circ} \mathrm{C}\right)$ lorsque les variations de température par unité de temps sont différentes (respectivement $3{ }^{\circ} \mathrm{C} / \mathrm{h}$ et $1,5^{\circ} \mathrm{C} / \mathrm{h}$ ) et ces conclusions sont systématiquement vraies quelle que soit la profondeur considérée.

2) Une conclusion du même type peut être obtenue par l'analyse qualitative simultanée des profils thermiques et des profils de potentiel (fig. 12). Pour les zones situćes en profondeur (de $-10 \mathrm{~cm}$ à $-25 \mathrm{~cm}$ ), bien que les gradients thermiques aux différentes heures du jour soient bien contrastés, les réponses psychrométriques sont du même ordre de grandeur ( $\sim 1$ bar). Inversement, bien que les profils thermiques près de la surface (de 0 à $-5 \mathrm{~cm}$ ) restent proches cntre le milieu de la journée et le soir, les profils de potentiel sont contrastés. Ici encore, il apparaît que la mesure du potentiel ne peut être directement assimilée à une mesure de température ou de gradient de température.

3) L'évolution progressive du dessèchement des horizons au cours du temps est bien marquéc: la position des maximums augmente systématiquement d'un jour à l'autre (fig. 10) et l'évolution progressive du dessèchement du sol se superpose ainsi à l'évolution des valeurs de potentiel à l'échelle de la journée. Ce dessèchement apparent du sol ne peut d'ailleurs être imputé au réchauffement progressif des horizons, de plus en plus secs d'un jour à l'autre : ainsi, à $-1 \mathrm{~cm}$, la valeur du potentiel passe de $-21,6$ bars à $42,6^{\circ} \mathrm{C}$ (jour 1 à $14 \mathrm{~h} 15$ ) à moins de -80 bars à $41,7^{\circ} \mathrm{C}$ (jour 2 à $14 \mathrm{~h} \mathrm{15)}$ : il y a bien ici un marquage de l'évolution réelle des couches de surface.

Par contre, les mesures indiquent un potentiel nul ou quasiment nul durant la nuit. Il y a donc un contraste « journuit " considérable, qui correspond probablement à des phases de condensation nocturne dans les horizons les plus froids, y compris lorsque le dessèchement est déjà prononcé. Dans ce cas, il y a saturation ou quasi-saturation de la phase gazeuse sans que l'ensemble « solide + liquide " soit réhumecté totalement dans le même laps de temps.

Au total, ces observations ne permettent pas de conclure que la non-isothermie n'induit pas de biais dans la mesure

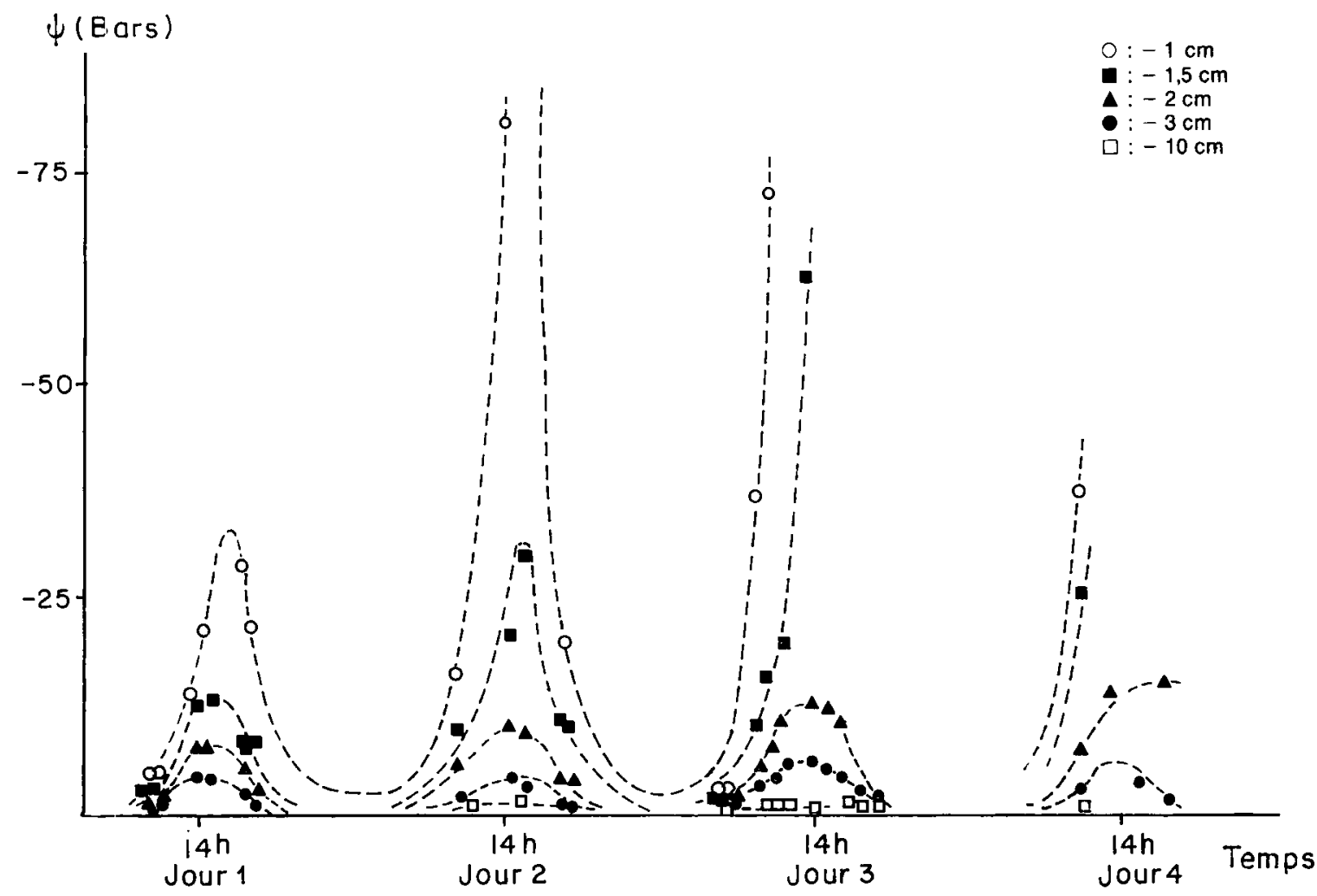

Figure 10

Potentiel hydrique mesuré in situ en fonction du temps sous sol nu. 


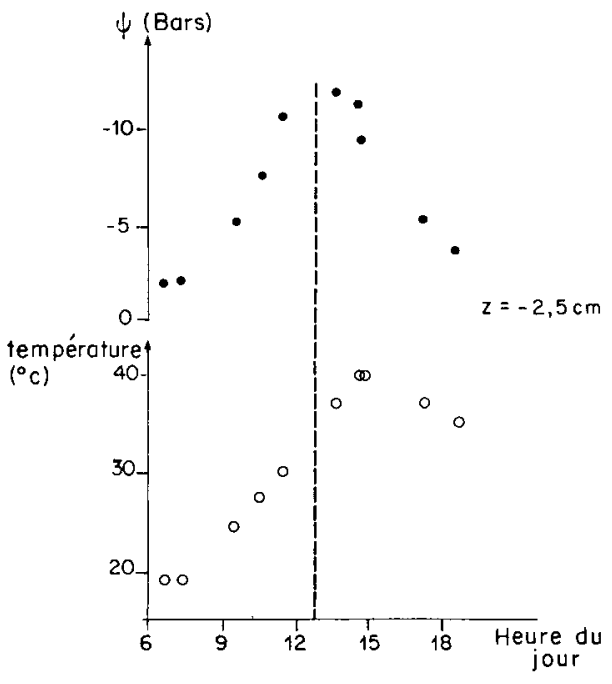

Figure 11

Potentiel hydrique et température du sol en fonction de l'heure du jour.

Soil water potential and temperature versus time of day.

psychrométrique, mais elles indiquent que la mesure psychrométrique ne peut pas être directement assimilée à une mesure de température. Il concient donc de procéder à une analyse exhaustive des sources d'erreurs possibles en psychrométrie liées à la non isothermie.

\section{ANALYSE DES SOURCES D'ERREURS LIÉES A LA NON-ISOTHERMIE}

D'une façon générale, les sources d'erreurs liées à la nonisothermie peuvent être classées en 2 grandes catégories :

- D'une part, le fonctionnement du psychromètre est perturbé par les variations de température :

- dans le temps : il y a dérive de la température de la jonction de référence pendant la mesure (DURAND, 1970) ;
- dans l'espace : les gradients thermiques verticaux induisant une f.e.m. non nulle même lorsque la soudure est sèche, f.e.m. normalement annulée par la mise à zéro électrique du circuit juste avant la mesure.

- D'autre part, l'existence d'un régime thermique variable dans le sol pose le problème de l'équilibre thermodynamique entre l'atmosphère de la capsule et le sol, problème qui fait notamment intervenir la perméabilité de la paroi poreuse utilisée.

\section{A. Analyse des effets thermiques proprement dits}

\section{Dispositif expérimental}

Un psychromètre à céramique est plongé dans une solution à potentiel connu et l'ensemble est soumis à des variations thermiques (échauffement ou refroidissement). On a réalisé 3 manipulations :

- manipulation 1 : échauffement de la solution (20 à $25^{\circ} \mathrm{C}$ ),

- manipulation 2 : refroidissement de la solution (25 à $20^{\circ} \mathrm{C}$ ),

- manipulation 3: échauffement de la solution (20 à $25^{\circ} \mathrm{C}$ ), mais en laissant dériver le «zéro électrique »du circuit afin d'estimer la différence de température entre la soudure de référence et la soudure de mesure.

L'avantage lié à l'utilisation d'un psychromètre à céramique plongé dans la solution réside dans le fait que la solution affleure sur la paroi interne de la capsule psychrométrique et que les problèmes éventuels de barrière de diffusion sont ainsi totalement éliminés.

\section{Résultats expérimentaux}

Le tableau 6 donne les résultats des manipulations 1 et 2 (échauffement ou refroidissement) pour les variations thermiques compatibles avec celles observées in situ. Quelle que soit la méthode de mesure, les valeurs observées restent très proches de la valeur théorique du potentiel de la solution. En moyenne, les écarts observés sont de plus ou moins 1 bar, c'est-à-dire de l'ordre de grandeur de la précision de

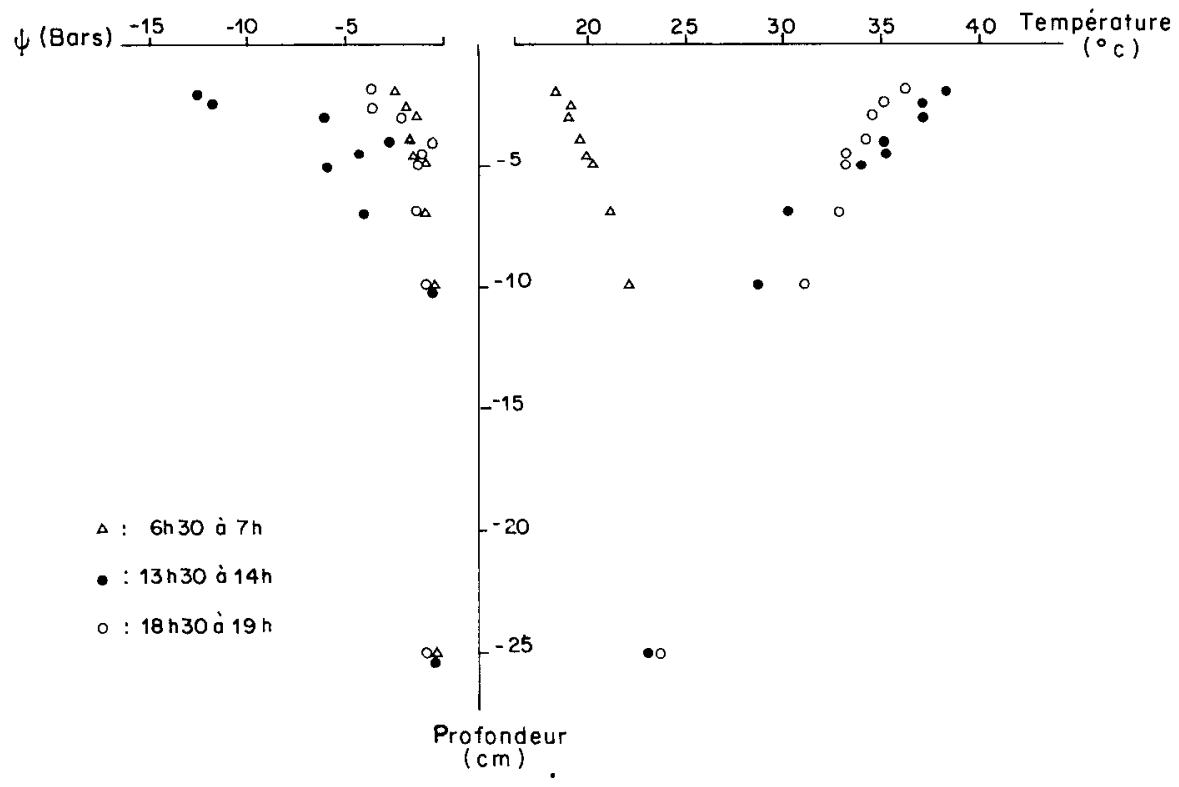

Figure 12 
la mesure elle-même : la variation de températurc dans le temps n'induit donc que des perturbations négligeables ou nulles.

Le tableau 7 donne une estimation des différences de température eritre les jonctions du thermocouple à partir de l'enregistrement de la dérive du «zéro électrique », pour une manipulation analogue, par ailleurs, à la manipulation 1. On exclut donc le cas où le dispositif expérimental choisi ne reproduirait, par échauffement ou refroidissement, qu'une succession d'états parfaitement isothermes et infiniment proches les uns des autres. L'échauffement différentiel des éléments du psychromètre induit une différence de température entre la soudure de mesure et celle de référence qui peut être supérieure à $1 / 10^{\circ} \mathrm{C}$ (tabl. 7 ), ce qui est important dans le cadre de la technique psychrométrique (cf. tabl. 5). Malgré l'induction de ces différences de température, l'écart moyen entre les valeurs mesurées et théoriques est égal à 1 bar, comme pour les résultats du tableau 6. De plus, les gradients thermiques induits par l'échauffement du bain sont d'un ordre de grandeur compatible avec ceux qui peuvent exister dans une couche de sol à fort gradient thermique ; ainsi l'observation de la figure 12 permet de montrer que, dans un cas où la température du sol est de $38,3^{\circ} \mathrm{C}$ à $-2 \mathrm{~cm}(13 \mathrm{~h} 30)$, les gradients thermiques expérimentaux observés s'étalent de $0,146^{\circ} \mathrm{C} / \mathrm{mm}$ à $-2 \mathrm{~cm}$ à $0,110^{\circ} \mathrm{C} / \mathrm{mm}$ à $-10 \mathrm{~cm}$.

Au total, dans la gamme de non-isothermie imposée, la mesure psychrométrique n'est pas perturbée de plus ou moins 1 bar par l'échauffement ou le refroidissement, même dans le cas où ces variations de température dans le temps cœxistent avec des gradients de température dans l'espacc.

\section{TABLEAU 6}

Mesures psychrométriques avec échauffement ou refroidissement du milieu (potentiel théorique $=-40,8$ bars à $20{ }^{\circ} \mathrm{C}$ ). Psychrometric measurements with both increasing or decreasing temperature (theoretical water potential $=-40.8$ bar at $20^{\circ} \mathrm{C}$ ).

\begin{tabular}{|c|c|c|c|c|c|c|c|c|c|c|c|c|c|c|}
\hline Température $\left({ }^{\circ} \mathrm{C}\right)$ & 20,0 & 20,1 & 20,5 & 20,9 & 21,6 & 22,4 & 23,1 & 23,5 & 24,1 & 24,7 & 25,7 & 27,0 & 27,2 & \\
\hline Echauffement $\left({ }^{\circ} \mathrm{C} / \mathrm{h}\right)$ & \multicolumn{2}{|c|}{0,7} & & & & & 5,3 & & & \multicolumn{5}{|c|}{3,5} \\
\hline$|\psi|$ bar $\left({ }^{1}\right)$ & 38,6 & 38,6 & 39,7 & 39,6 & 39,5 & 39,7 & 40,1 & 40,5 & 40,1 & 40,4 & 41,1 & 40,5 & 40,7 & $\begin{array}{r}\mu=39,9 \\
\sigma=\quad 0,8\end{array}$ \\
\hline$|\psi|$ bar $\left(^{2}\right)$ & 40,0 & 40,1 & 40,0 & 39,4 & 39,5 & 39,5 & 39,5 & 40,0 & 39,0 & 39,0 & 39,9 & 39,1 & 38,6 & $\begin{array}{l}\mu=39,4 \\
\sigma=0,4\end{array}$ \\
\hline
\end{tabular}

\begin{tabular}{lccccccccccccc}
\hline Température $\left({ }^{\circ} \mathrm{C}\right)$ & 25,2 & 25,1 & 25,0 & 24,6 & 24,3 & 23,8 & 23,3 & 22,8 & 22,3 & 21,0 & 20,7 & \\
\hline Refroidissement $\left({ }^{\circ} \mathrm{C} / \mathrm{h}\right)$ & & 0,8 & & & & & 4,4 & & & 3,1 & \\
\hline$|\psi|$ bars $\left({ }^{1}\right)$ & 41,2 & 42,6 & 42,3 & 42,4 & 42,2 & 42,7 & 42,2 & 41,4 & 41,4 & 41,7 & 40,4 & $\mu=41,9$ & $\sigma=0,7$ \\
\hline$|\psi|$ bar $\left({ }^{2}\right)$ & 41,0 & 41,3 & 41,0 & 41,0 & 40,7 & 41,2 & 40,8 & 40,0 & 40,0 & 41,1 & 38,9 & $\mu=40,7$ & $\sigma=0,7$ \\
\hline
\end{tabular}

(1) Méthode du point de rosée. $\left({ }^{2}\right)$ Méthode classique.

TABLEAU 7

Différences de température entre les soudures de mesure et de réfërence en phase d'échauffement du milieu ambiant. Temperature differences between reference and measurement junctions with increasing temperature all around the psychrometer.

\begin{tabular}{|c|c|c|c|c|c|c|c|c|}
\hline Température $\left({ }^{\circ} \mathrm{C}\right)$ & 20,1 & 20,6 & 21,9 & 22,8 & 23,8 & 24,1 & 24,9 & \\
\hline Echauffement $\left({ }^{\circ} \mathrm{C} / \mathrm{h}\right)$ & 0,7 & 3,8 & 6,0 & 6,0 & 6,0 & 4,1 & 4,1 & \\
\hline Dérive du «zéro électrique » $(\mu \mathrm{V})$ & 3,3 & 5,1 & 6,8 & 6,7 & 6,9 & 7,0 & 4,8 & \\
\hline $\begin{array}{l}\text { Différence de ternpérature entre les } \\
\text { soudures }\left({ }^{\circ} \mathrm{C}\right)\end{array}$ & 0,055 & 0,086 & 0,112 & 0,111 & 0,114 & 0,116 & 0,080 & \\
\hline$|\psi|$ mesuré (bar) & 38,8 & 43,3 & 42,3 & 43,3 & 42,9 & 42,8 & 41,8 & $\begin{array}{l}\mu=42,2 \\
\sigma=1,6\end{array}$ \\
\hline$|\psi|$ théorique (ba:) & 40,8 & 40,9 & 41,1 & 41,2 & 41,4 & 41,5 & 41,6 & $\begin{array}{l}\mu=41,2 \\
\sigma=0,3\end{array}$ \\
\hline
\end{tabular}




\section{B. Rôle de la capsule poreuse vis-à-vis de la diffusion gazeuse}

\section{Dispositif expérimental}

Trois manipulations sont réalisées en disposant les psychromètres dans une phase gazeuse $\left(\sim 100 \mathrm{~cm}^{3}\right)$ au-dessus d'une solution à potentiel connu. Dans les 3 cas, on soumet le dispositif à un échauffement intense $\left(>5^{\circ} \mathrm{C} / \mathrm{h}\right)$.

Manipulation $5:$ thermocouple nu, sans capsule poreuse, placé dans une phase gazeuse échauffé de 20 à $27^{\circ} \mathrm{C}$.

Manipulation 5:thermocouple nu plus thermocouple avec grille, placés dans une phase gazeuse échauffée de 20 à $25^{\circ} \mathrm{C}$.

Manipulation 6 : thermocouple nu plus thermocouple avec céramique, placés dans une phase gazeuse échauffée de 20 à $30^{\circ} \mathrm{C}$.

\section{Résultats expérimentaux}

En partant d'une situation initiale de 40,8 bars à $20^{\circ} \mathrm{C}$, si la vapeur d'eau ne transitait absolument pas à travers les parois poreuses (grille ou céramique), la diminution théorique de $\mathrm{p} / \mathrm{p}_{\mathrm{o}}(\mathrm{T})$ devrait conduire à une valeur absolue de potentiel supérieure à 80 bars dès $20,5^{\circ} \mathrm{C}$. Dans ce cas, on devrait très rapidement accéder à des valeurs de potentiel situées hors de la gamme de mesure psychrométrique (70 à 80 bars). De même, tout caractère limitant de la capsule poreuse vis-à-vis de la diffusion devrait se traduire par une augmentation permanente de la valeur absolue du potentiel.

Les manipulations 4, 5, 6 étant réalisées dans des conditions thermiques différentes, nous avons normalisé les données afin de comparer plus facilement les 3 manipulations: soit $X_{i}$ une valeur de potentiel lue à un moment quelconque, pour un psychromètre et une manipulation (4, 5 ou 6). On remplacera la séries des $X_{i}$ par la série des $Y_{i}$ avec

$$
\begin{gathered}
Y_{i}=X_{i} / \bar{X}_{i} \text {, en posant } \\
\bar{X}_{i}=\frac{1}{n} \sum_{i=1}^{n} X_{i}
\end{gathered}
$$

La figure 13 donne les distributions de fréquences des valeurs $Y_{i}$ dans le cas des thermocouples nus, à grille ou à céramique : globalement et quel que soit le cas expérimental analysé, les fluctuations des réponses psychrométriques sont faibles et normales. On constate que, dans le cas où le thermocouple est protégé par une capsule poreuse, les fluctuations sont comparables aux fluctuations aléatoires du thermocouple nu $(\mathrm{CV}=3,5$ à 5 p. 100). Non seulement chaque point mesuré reste toujours dans la gamme de mesure psychrométrique même pour des échauffements $>5^{\circ} \mathrm{C} / \mathrm{h}$, mais aucune augmentation progressive de la valeur absolue de la mesure n'est mise en évidence.

La présence de la capsule poreuse, quelle que soit sa nature, ne semble donc pas induire de perturbation de la mesure dans la gamme des amplitudes thermiques testées. L'ensemble de ces conclusions est d'ailleurs compatible avec celles de VALANCOGNE \& DAUDET (1974) sur les problèmes thermiques et celles de RAWLins \& DalTon (1967) sur l'ensemble des aspects diffusifs et thermiques en psychrométrie.
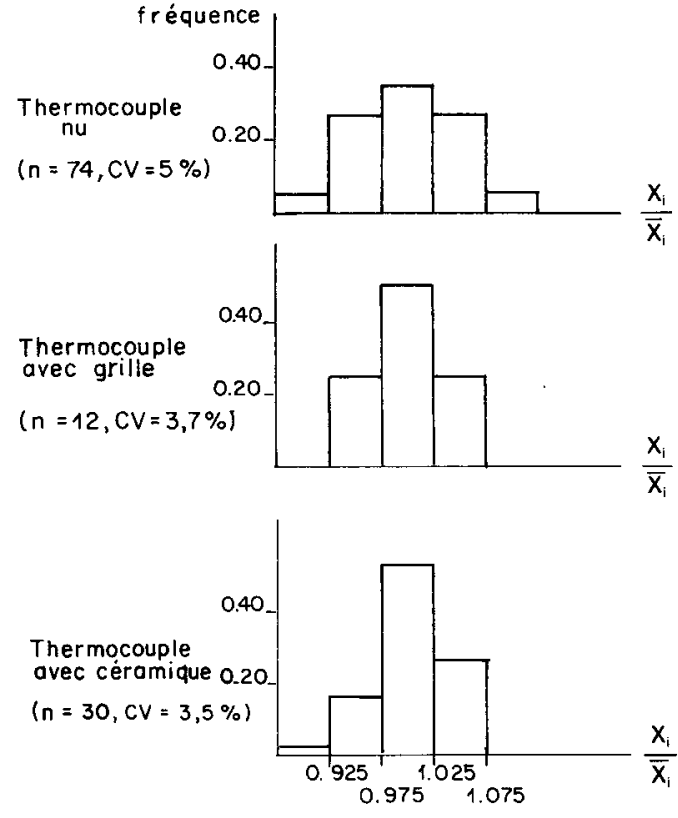

Figure 13

Effet de la nature de la capsule sur la réponse psychrométrique. Histogramme des valeurs $X: \bar{X}_{\text {. }}$.

$\left(X_{i}\right.$ : potentiel hydrique mesuré au temps $i$.

$\bar{X}_{i}:$ potentiel hydrique moyen pendant la durée de l'expérience.

Effect of the ceramic or steel shield on the psychrometric measurements.

Histogram of the $X / \bar{X}_{i}$ values.

$\left(X_{i}:\right.$ water potential measurement at time $i$.

$\bar{X}_{i}$ : mean water potential during the experiment.

\section{CONCLUSION}

L'analyse des mesures psychrométriques en laboratoire et in situ permet de dégager plusieurs points importants :

1. La réponse psychrométrique expérimentale en conditions isothermes est conforme à la prévision donnée par les modèles. Pour la méthode du point de rosée, il apparaît donc que la convergence vers le point de rosée est bien assurée, pourvu que la relation $\mathrm{U}_{\max }=\mathrm{f}(\mathrm{T})$ soit préalablement connue expérimentalement. Pour la méthode classique, l'adéquation entre les prévisions et les résultats indique que les paramètres géométriques des psychromètres obtenus auprès du constructeur sont fiables et que la fabrication technique des psychromètres est homogène puisque tous les psychromètres correspondent à un étalonnage pratiquement unique.

Pour les 2 méthodes, il est donc envisageable de limiter considérablement, voire supprimer, les procédures d'étalonnage.

2. L'analyse expérimentale détaillée des sources d'erreurs induites par la non-isothermie et l'analyse qualitative des résultats obtenus in situ indiquent que cette situation n'entraîne que des perturbations négligeables visà-vis de la mesure. Les mesures obtenues in situ au voisinage de la surface doivent donc rendre compte, pour une très large part, des variations réelles du potentiel de l'eau de la phase gazeuse.

3. Globalement, la technique psychrométrique apparaît utilisable dans une large gamme de situations expérimentales, sa plus importante limitation résultant dans la précision 
de la mesure proprement dite. Elle constitue ainsi un atout important en physique du sol dans tous les cas où la tensiométrie n"est plus utilisable. En ce qui concerne lc comportement respectif des phases liquide et gazeuse, on conçoit que l'équilibre thermodynamique instantané de ces phases ne soit réalisé que lorsque la taille des agrégats devient infiniment petite. Dans le cas contraire, qui est aussi le cas général, il semble tout à fait envisageable que cet équilibre ne concerne que la zone la plus périphérique des agrégats. A cet égard, l'utilisation des modèles de transfert de masse et de chaleur dans les sols, qui suppose l'équilibre instantané des phases indépendamment de la taille des éléments structuraux, pose un problème théorique ct pratique important.

Reçu le 21 février 1983. Accepté le 19 septembre 1983.

\section{RÉFÉRENCES BIBLIOGRAPHIQUES}

Durand R., 1970. Utilisation des thermocouples. 131-141. In: Techniques d'étude des facteurs physiques de la biosphère. I.N.R.A., Paris, 544 p.

Kyte J. R., Madden A. J., Piret E. L., 1953. Natural convection heat transfer at reduced pressure. Chem. Eng. Progr., 49, 653-662. Lang A. R. G., 1967. Osmotic coefficients and water potentials of sodium chloride solutions from 0 to $40^{\circ} \mathrm{C}$. Aust. J. Chem., 20, 20172023.

Merril S. D., Rawlins S. L., 1972. Field measurement of soil water potential with thermocouple psychrometers. Soil Sci., 113, 2, 102109.

Millar B. D., 1971a. Improved thermocouple psychrometer for the measurement of plant and soil water potential. I. Thermocouple psychrometry and an improved instrument design. J. Exp. Bot., 22, 73, 875-890.

Millar B. D., 1971b. Improved thermocouple psychrometer for the measurement of plant and soil water potential. II. Operation and calibration. J. Exp. Bot., 22, 73, 891-905.

Millar B. D., 19\%4. Improved thermocouple psychrometer for the measurement of plant and soil water potential. III. Equilibration. $J$. Exp. Bot., 25, 89, 1070-1084.

Monteith J. L., Owen P. C., 1958. A thermocouple method for measuring relative humidity in the range $95-100 \%$. J. Sci. Instrum., 35, 443-446.
Peck A. J., 1968. Theory of the Spanner psychrometer. 1. The thermocouple. Agric. Meteorol., 5, 433-447.

Peck A. J., 1969. Theory of the Spanner psychrometer, 2. Sample effects and equilibration. Agric. Meteorol., 6, 111-124.

Rawlins S. L., 1966. Theory for thermocouple psychrometers used to measure water potential in soil and plant samples. Agric. Meteorol., 3, 293-310.

Rawlins S. L., Dalton F. N., 1967. Psychrometric measurement of soil water potential without precise temperature control. Soil Sci. Soc. Am. Proc., 31, 297-301.

Scotter D. R., 1972. The theoretical and experimental behaviour of a Spanner psychrometer. Agric. Meteorol., 10, 125-136.

Slack D. C., Riggle F. R., 1980. Effects of Joule heating on thermocouple psychrometer water potential determinations. Am. Soc. Agric. Eng., 23, 1, 877-883.

Spanner D. C., 1951. The Peltier effects and its use in the measurement of suction pressure. J. Exp. Bot., 2, 145-168.

Valancogne C., Daudet F. A., 1974. Adaptation de la technique micropsychrométrique à l'enregistrement du potentiel hydrique dans le sol en place: Problèmes posés par la température. Ann. Agron., 25 (5), 733-751. 